\title{
Pompes primaires sodium à grandes dimensions
}

\section{oêl Guidez}

CEN/Cadarache-Chargé d'affaires des pompes des réacteurs rapides

\section{Richard Canavelis el Pierre Guilloiseau}

BERGERON S. A.

\section{Introduction}

Le but de cet exposé est de présenter un travail de conception effectué au niveau des pompes primaires sodium des réacteurs rapides.

Ce travail a été réalisé dans le cadre d'un réacteur 1200 MWe, 4 pompes et avec le soutien de plusieurs industriels (Bergeron - Snecma-Neyrpic).

Il a abouti à la conception et au dessin d'une pompe de type nouveau qui pourra être étudiée en vue d'une réalisation éventuelle pour la filière des réacteurs rapides.

\section{Méthode de travail}

Schéma hydraulique du réacteur

Le rôle de la pompe primaire dans un réacteur rapide est de propulser le sodium à travers le cœur de ce réacteur (voir fig. 10).

Le fluide est aspiré dans le collecteur froid puis refoulé à travers le cœur dans le collecteur chaud. Il traverse ensuite les échangeurs intermédiaires pour revenir dans le collecteur froid. La pompe primaire est ici une pompe mécanique fonctionnant sous niveau libre de sodium.

\section{But de l'étude}

Le but de l'étude était d'effectuer un examen des différentes solutions hydrauliquement envisageables pour concevoir une pompe de diamètre minimal de manière à:

- diminuer les dimensions, donc le poids et le prix de cette pompe ;

- permettre une diminution du diamètre du bloc réacteur.
Définition des paramètres externes et internes

La conception d'une pompe résulte du choix de deux sortes de paramètres.

\section{- Les paramètres externes}

Ce sont les paramètres imposés par le reste du réacteur et auxquels doit se plier le concepteur de la pompe.

On citera en particulier :

- nombre de pompes,

- débit cœur,

- débit refroidissement,

-... perte de charge globale du circuit (début de cycle et fin de cycle),

- choix du débit maximal,

-- courbes de perte de charge maxi et mini,

- choix de la vitesse maxi.

- pressurisation du ciel de pile, régime nominal,

- niveau NA, collecteur froid, régime nominal,

- hauteur de cuve, forme du fond de cuve, axe de la pompe,

- puissance retenue lors du fonctionnement à $n-1$ pompes sur $n$

- procédure de fonctionnement retenue lors de la perte d'une pompe,

- marge sur la cavitation (critères de bruit et de fiabilité)

- accidents retenus (séisme, surpression),

- état de surface exigé (décontamination),

- hydraulique collecteur froid,

- hydraulique du sommier,

- température maximale exigée en fonctionnement normal. 
exemple:

La définition de la hauteur de cuve de la forme du fond de cuve de la position de l'axe pompe,

permet de caler la roue vis-à-vis du niveau sodium collecteur froid.

La connaissance du la pression dans le ciel de pile nous donnera alors le NPSF disponible.

\section{- Les paramètres internes}

Ce sont les choix technologiques internes à la pompe:

- type de veine hydraulique

- présence d'une jupe d'aspiration

- choix d'un système anti-dévirage

etc.

On peut dire que ces paramètres fixent la réponse $d u$ pompier au problème posé.

\section{Esprit de l'étude}

- On a gardé les paramètres externes de la pompe primaire Super Phenix.

En particulier:

-- même débit nominal : $4,76 \mathrm{~m}^{3} / \mathrm{s}$

- même hauteur nominale : $69,6 \mathrm{~m}$

- mêmes conditions géométriques.

On est donc toujours dans le cadre d'un 1200 MWe 4 pompes.

- On a effectué ensuite un balayage des paramètres internes, c'est-à-dire des choix technologiques possibles.

Le premier balayage à effectuer se situait au niveau du choix de type de veine hydraulique.

C'est ce balayage qui est présenté ici.

\section{Définition de la veine hydraulique}

\section{Différents types de roue}

On peut envisager 5 solutions: (voir fig. 1):

- pompe double étage : aspiration haute

- pompe roue double aspiration basse

- pompe roue simple : aspiration haute aspiration basse

\section{Différents types de diffuseur}

\section{Volutes}

Des dessins ont été faits avec volute (fig. 2 par exemple). Mais les problèmes de supportage et d'encombrement font rejeter ce type de diffuseur.

\section{Problèmes de dénontabilité}

La notion de démontabilité de l'ensemble de l'organe de récupération ne pose pas de problème particulier pour les pompes à aspiration haute.

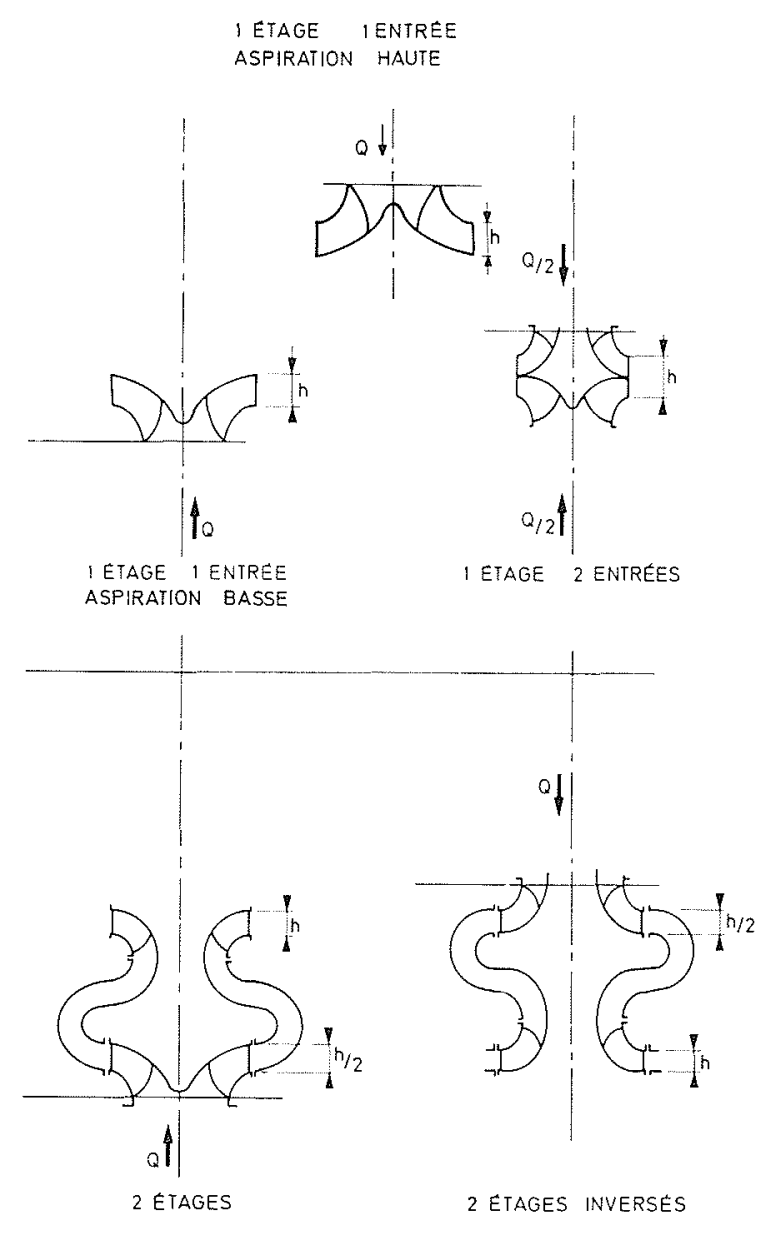

ligure 1 - Différents types de rove

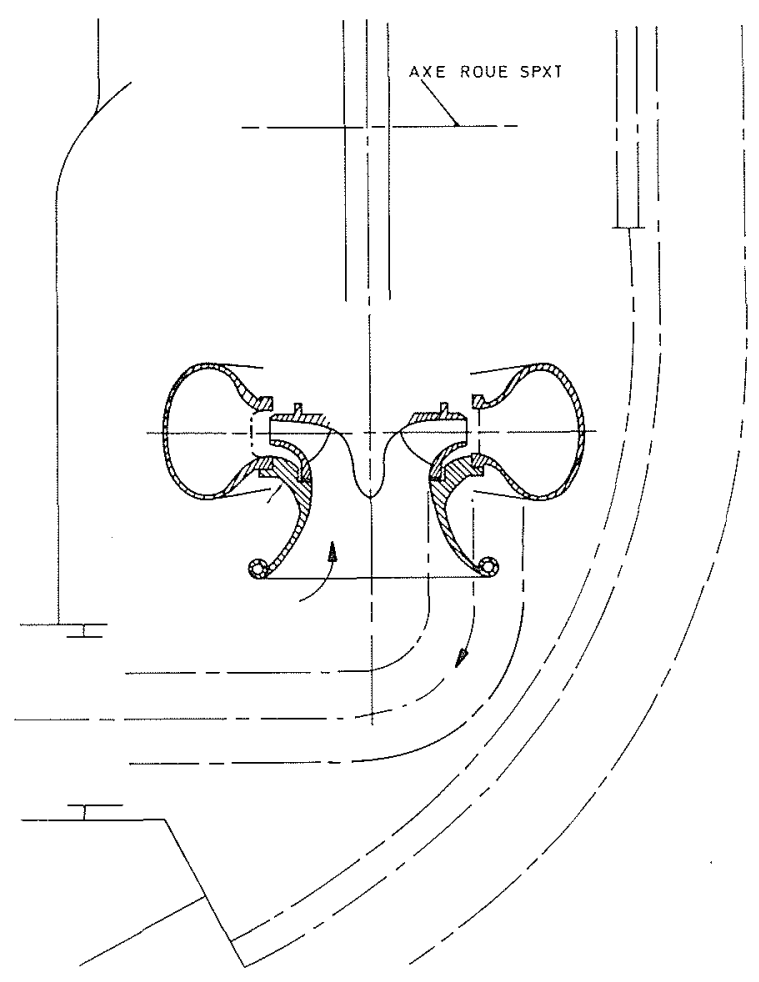

Figure 2 - Pompe avec volute 


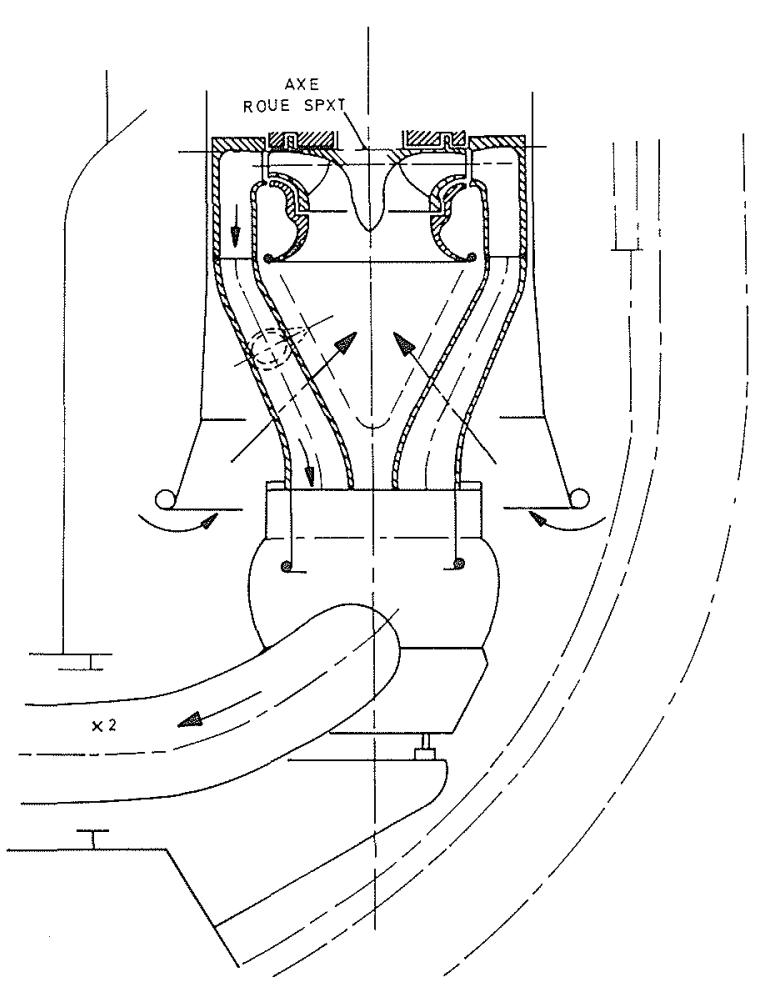

Figure 3 - Pompe à flux croisés

Par contre pour les pompes à aspiration basse, il se pose un problème dû au fait que le système d'étanchéité entre la partie démontable et la partie fixe nous conduira alors automatiquement à des solutions flux croisés (voir figures 3 et 4 ).

Les solutions flux croisés ayant été rejetées (pour des problèmes de complexité hydraulique de l'ensemble, et en particulier dans les relations de la pompe vis-à-vis du collecteur froid), toutes les solutions à aspiration basse seront dotées de collecteurs non démontables.

\section{Solutions restantes}

Il reste alors plusieurs solutions :

- pompe deux étages, aspiration par le bas, collecteur non démontable,

- pompe deux étages, aspiration par le haut, collecteur démontable,

- pompe deux étages, aspiration par le haut, collecteur non démontable,

- pompe roue double, collecteur non démontable,

- pompe aspiration haute, collecteur fixe,

- pompe aspiration haute, collecteur démontable,

- pompe aspiration basse, collecteur fixe.

\section{Examen de la pompe double étage}

Aspiration par le bas-.. Collecteur fixe (voir fig. 5).

L'idée de base était de voir si le fait de diminuer la hauteur à fournir par la roue pouvait conduire à des diminutions importantes du diamètre de l'ensemble.

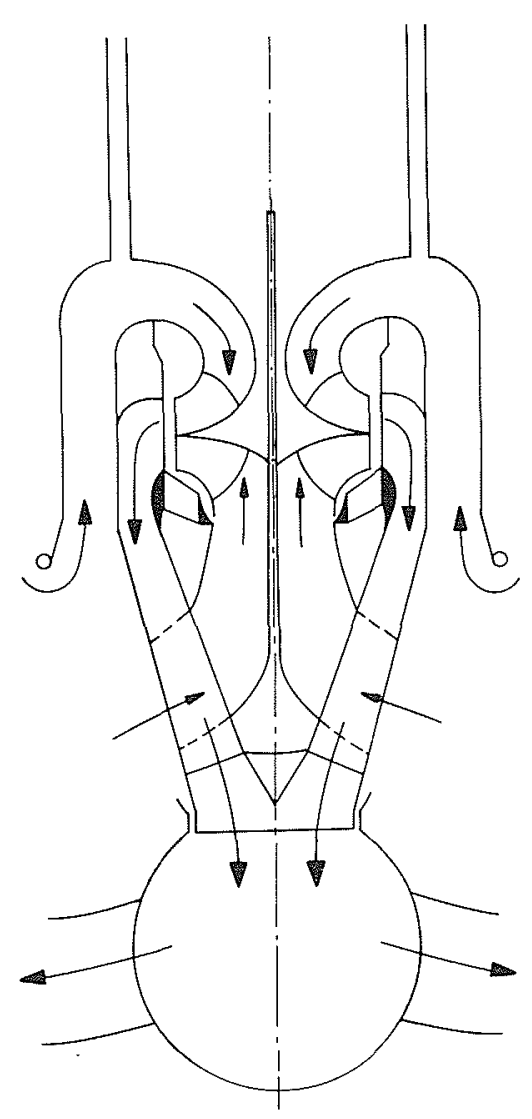

Figure 4 - Pompe à flux croisés

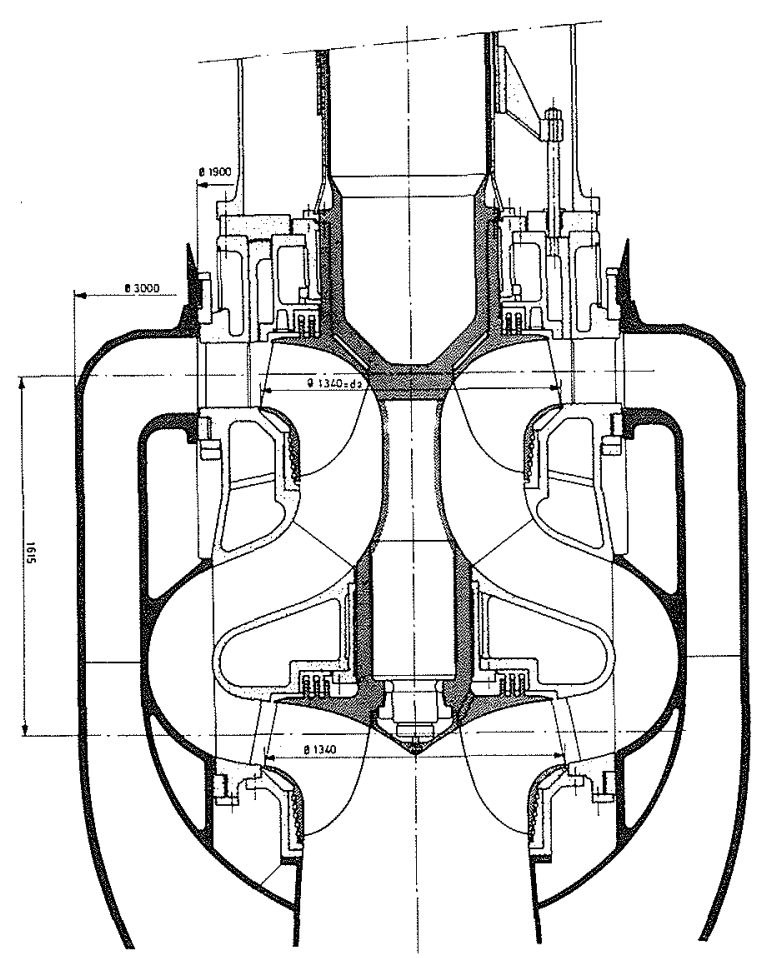

Figure 5 - Pompes double étage aspiration basse collecteur fixe 
Le dimensionnement présenté sur la figure 5 a été effectué en supposant que chaque roue crée $50 \%$ de la hau teur demandée.

NB : Ceci n'est donc pas le dimensionnement optimal de l'appareil qui serait obtenu en donnant à la première roue un rôle de pompe de gavage.

En fait, la présence des canaux de retour de la première roue à la deuxième annule les gains que l'on pouvait espérer ; or, de plus la solution présentait de nombreuses complications constructives :

- problèmes d'étanchéité à divers niveaux,

- nécessité de deux paliers en sodium ou d'un arbre hypercritique,

- complication importante de l'ensemble (et donc prix plus élevé).

Elle a donc été abandonnée.

Aspiration haute (collecteur fixe ou démontable).

Abandonnée pour les mêmes raisons.

\section{Examen de la pompe roue double (collecteur fixe)}

Cette solution présentait de nets avantages dimensionnels (voir fig. 6);

C'est en effet dans ce cas que l'on aura le plus faible diamètre de roue et donc le plus faible diamètre de partie démontable.

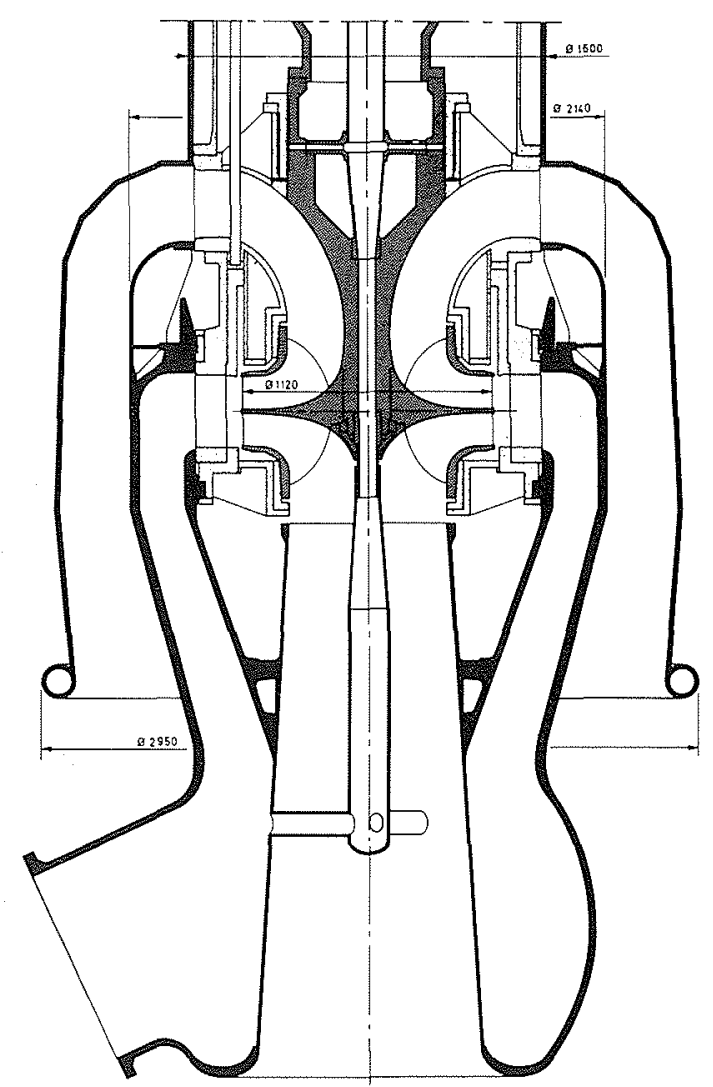

ligure 6 - Pompes roue double aspiration basse collecteur fixe
Mais la vitesse de rotation étant multipliée par 1,4 environ, il devenait indispensable de recourir à un arbre hypercritique ou à 3 paliers.

- La roue double faisait un porte à faux important.

- L’hydraulique générale d'ensemble était plus complexe.

- L'encombrement de la partie statorique était plus grand que celui de la pompe roue simple, aspiration basse, par suite de l'existence d'une jupe d'aspiration. Comme le gain dimensionnel sur la partie démontable était faible vis-à-vis de la solution retenue, cette solution a donc été abandonnée.

\section{Examen de la pompe aspiration haute}

\section{Collecteur démontable}

C'est la pompe type Super Phenix (voir fig. 7). De par la forme de la veine hydraulique, on s'impose donc:

- l'existence d'une jupe (structure lourde dans le réacteur);

- un dimensionnement diamétral important fixé par cette jupe (en cas de suppression de cette jupe, il reste la nécessité de laisser la place suffisante au fluide pour monter vers la pompe).

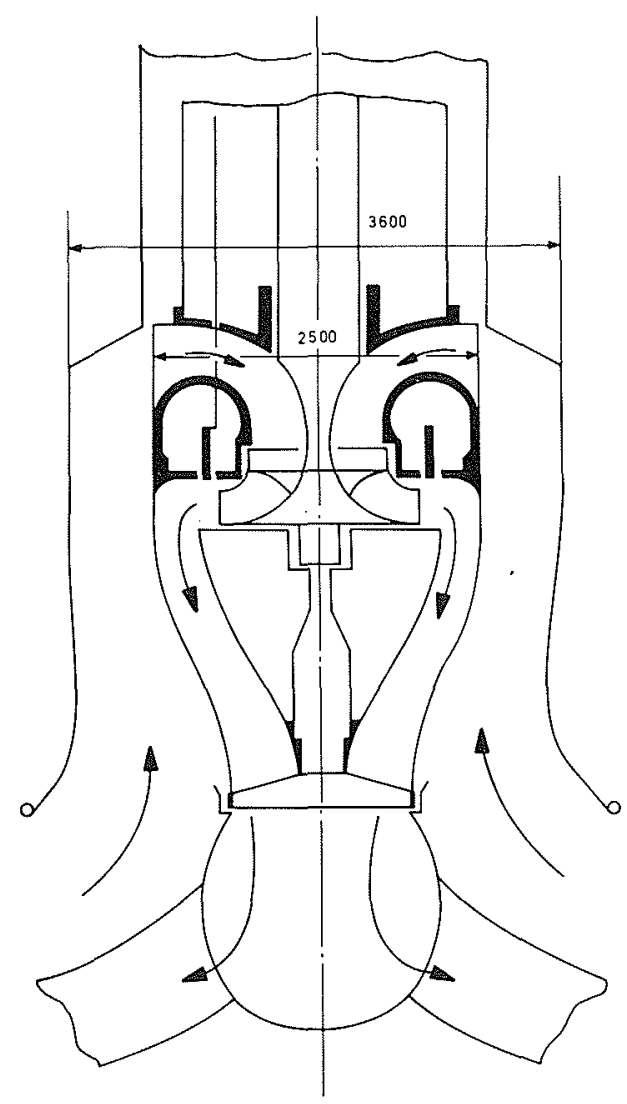

ligure 7 - Pompe roue simple aspiration haute collecteur démontable 


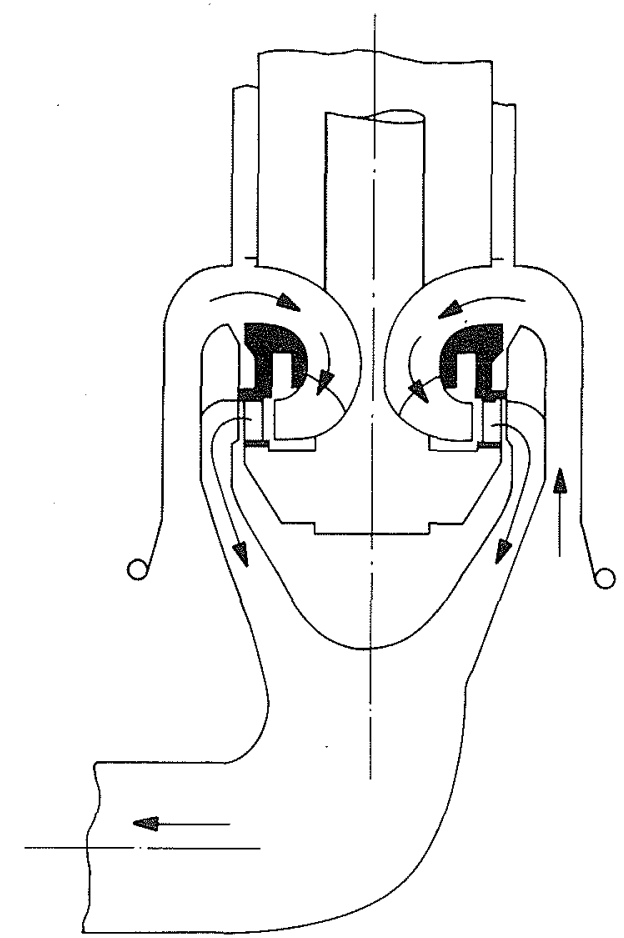

Figure 8 - Pompe roue simple aspiration haute collecteur fixe

\section{Collecteur fixe (figure 8)}

La solution à aspiration basse est alors préférable à tout point de vue.

\section{Examen de la pompe aspiration basse}

(Voir fig. 9)

- la solution présente des avantages dimensionnels importants :

$\phi$ démontable 1930 au lieu de 2470 (pompe SPX) $\phi$ partie fixe 2550 au lieu de 3600 (jupe d'aspiration SPX)

- elle présente une grande simplicité d'ensemble

- elle permet de garder une pivoterie deux paliers avec un arbre de diamètre raisonnable

- il y a beaucoup moins de pièces de fonderie (pas de lanterne d'aspiration en particulier)

- elle permet la suppression d'une structure : la jupe d'aspiration

- les gains dimensionnels conduisent à un gain de poids, d'environ $30 \%$ sur la pompe.

\section{Conclusion}

La solution de plus petit diamètre était la solution roue double.

Mais le gain dimensionnel était faible vis à vis de la solution aspiration axiale basse.

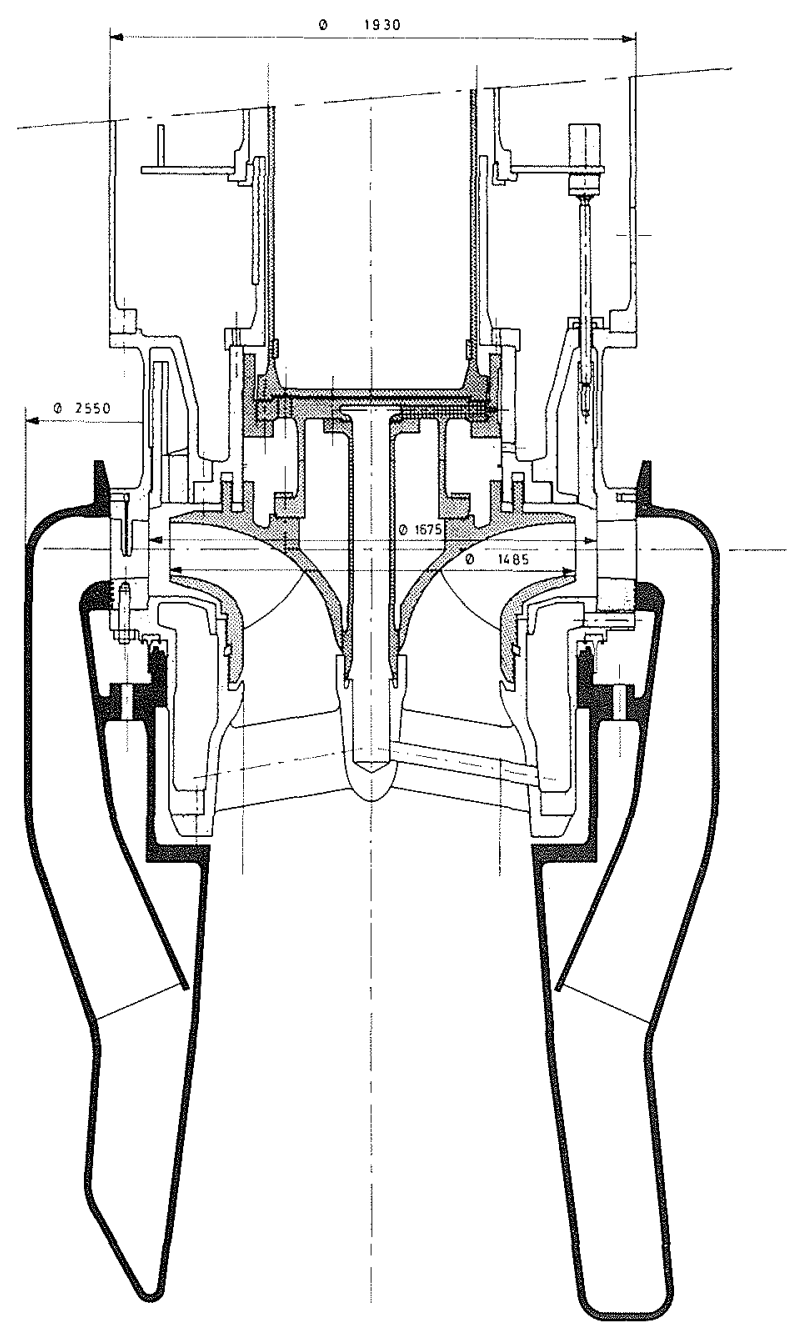

Figure 9 - Pompe roue simple aspiration basse collecteur fixe

Comme celle-ci présentait l'avantage d'une plus grande simplicité et d'une plus grande continuité technologique (en particulier, vis à vis de la pivoterie) on s'oriente donc vers la poursuite de l'étude de ce dernier modèle.

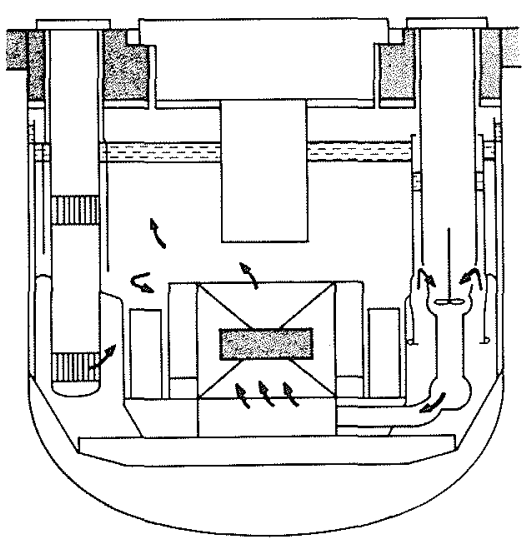

Figure 10-Schéma du bloc réacteur. 


\section{Quelques problèmes hydrauliques liés à la solution retenue}

\section{Existence de pré-aubes démontables}

$\mathrm{Vu}$ les déplacements horizontaux du collecteur froid, il sera nécessaire pour des raisons de jeux au labyrinthe inférieur que la partie statorique de ces labyrinthes soit reliée à la partie démontable de la pompe. Ceci conduit donc à traverser le flux à la sortie de la roue par un ensęmble de pré-aubes reliées à la partie démontable de la pompe.

\section{Alimentation du palier}

Elle est effectuée comme pour Super Phenix par une alimentation centrale qui permet de récupérer un supplément de pression par centrifugation.

L'ensemble du système d'alimentation est démontable.
Choix de la partie fixe de l'organe de récupération

Il apparaît trois possibilités :

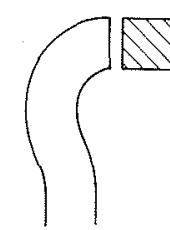

(1)

pas d'aube dons la pièce fixe

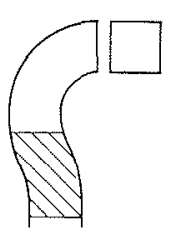

(2)

aubes dans to pièce fixe mais pas dons le coude

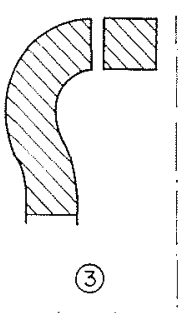

aubes dans lo pièce fixe
Le tableau suivant récapitule les avantages et inconvénients respectifs des 3 solutions.

\begin{tabular}{|c|c|c|c|}
\hline & Solution (1) & Solution (2) & Solution (3) \\
\hline Construction & mécano soudé & mécano soudé & fonderie \\
\hline Rendement hydraulique & moins $5 \%$ inférieur & \multicolumn{2}{|c|}{ identique } \\
\hline Pulsations de pression & risques & & \\
\hline $\begin{array}{l}\text { Entrée dans les tuyau- } \\
\text { teries de refoulement }\end{array}$ & $\begin{array}{l}\text { Composante circulaire } \\
\text { de rotation résiduelle }\end{array}$ & \multicolumn{2}{|c|}{ peu de problème } \\
\hline Comparaison des formes & $\begin{array}{l}\text { la forme la plus } \\
\text { volumineuse en } \phi\end{array}$ & $\begin{array}{l}\text { La forme la moins } \\
\text { volumineuse en } \phi\end{array}$ & $\begin{array}{c}\text { presque identique } \\
\text { au (2) }\end{array}$ \\
\hline Inspectabilité & $\begin{array}{c}\text { Toutes les aubes } \\
\text { inspectables }\end{array}$ & \multicolumn{2}{|c|}{$\begin{array}{c}\text { Les aubes les plus sollicitées } \\
\text { sont inspectables }\end{array}$} \\
\hline $\begin{array}{l}\text { Décollement entre } \\
\text { aubes et pré-aubes }\end{array}$ & non & non & Risques potentiel \\
\hline
\end{tabular}

C'est donc la solution (2) qui a été retenue.

\section{Condusion}

Cette étude de conception a permis de déterminer une pompe optimisée vis à vis des paramètres externes imposés par le bloc-réacteur.
Cette pompe permet des gains dimensionnels importants tout en gardant une grande continuité technologique.

Des essais sur maquette au $1 / 10$ de cette pompe dans le collecteur froid sont actuellement en cours. 


\section{Discussion}

Président : M.P. BERGERON

M. le Président remercie M. GUIDEZ pour son exposé qui constitue une bonne synthèse de toutes les solutions, a priori envisageables, pour les pompes à sodium de grandes dimensions.

C'est là, dit-il, un travail utile car il permet d'éliminer un certain nombre de solutions que nos successeurs pourraient être amenés à envisager dans l'avenir. J'ouvre, sans plus tarder, la discussion.

Celle-ci début par un échange de vue entre MM. GUITON, CANAVELIS et GUIDEZ sur les raisons qui ont fait choisir le type de pompe adopté pour Super Phenix, type qui peut sembler s'éloigner de celui présenté lors de l'exposé.

Il en ressort que le choix pour Super Phenix de la pompe présentée comme optimale, d'un point de vue dimensionnel, n'aurait pas conduit pour le réacteur à un gain diamétral compte tenu des dimensions du bouchon tournant et des échangeurs (lesquelles déterminent pratiquement les dimensions de la cuve).
L'utilisation de la pompe présentée ne prend tout son intérêt que dans le contexte d'une série de choix effectués au niveau du réacteur qui permettent alors une diminution de diamètre de l'ensemble du bloc réacteur.

Se référant à la dernière diapositive projetéc par le conférencier, M. GILMER sollicite quelques précisions sur la possibilité de réduire encorc l'encombrement de la pompe.

Je ne comprends pas très bien, dit-il, pourquoi on aboutit à un diametre extérieur du bulbe aussi grand alors qu'habituellement, on part directement en diffuscur axial. S'il n'y avait pas les pré-diffuseurs - qui diffusent peu - on pourrait gagner énormément sur l'encombrement.

M. GUIDEZ rappelle que les pré-diffuscurs ont pour rôle principal de rendre les labyrinthes inférieurs solidaires de la partic démontable.

Il est par contre effectif que lcur rôle est faible au niveau de la diffusion et que, d'un seul point de vue hydraulique, on pourrait gagner en encombrement en partant directement en diffuseur axial.

\section{Abstract \\ Design of large sodium pumps}

The primary pumps of high-speed reactors operate under a free sodium surface level and deliver the fluid to the reactor core.

This report describes a design study of a sodium pump from the following angles:

a) Dimensions: Decreasing the diameter of a pump also decreases its weight (and therefore cost) and the diameter of the basic reactor unit.

b) Technology: The aim is to achieve a simple design whilst ensuring technological continuity of the pumps in the system.

The first design step was to determine reactor parameters to be considered in designing the pump. The most important of these are the following:

a) Rate of flow

b) Pressure drop across the reactor core,

c) Available NPSH (here: pressure at the top of the reactor + head of sodium between a rotor and the free sodium surface).

Design assumptions were $1200 \mathrm{MW}$, four pumps, and all corresponding external parameters.

The second step was to consider all potentially suitable hydraulic jet geometries associated with combinations of various rotor types (Fig. 1) and diffuser designs. The following were eliminated on initial analy sis:

a) Volute designs, as too bulky for a high-speed reactor.

b) Crossflow designs, as involving excessively difficult design and manufacturing problems.
This left the following designs for further consideration :

a) Two-stage pumps with bottom intakes and non-dismantleable manifolds.

b) Two-stage pumps with top intakes and non-dismantleable manifolds.

c) Two-stage pumps with top intakes and dismantleable manifolds.

d) Twin-rotor pumps with non-dismantleable manifolds.

e) High-level intake pumps with dismantleable manifolds.

f) High-level intake pumps with non-dismantleable manifolds.

g) Low-level intake pumps with non-dismantleable manifolds.

These designs were analysed, with the following results:

a) Two-stage designs are very complicated and do not result in any substantial improvement as regards geometry. Hence, they were eliminated.

b) Though twin-rotor designs are attractive as regards geometry, their bearings, overhung rotors and critical shaft speeds are major problems. They were therefore climinated.

c) High-level intake pumps require the largest amount of space and, in addition, have to be provided with an intake skirt. This resulted in their elimination.

d) Axial low-level intake pumps (lig. 9) combine simple design with optimal diameter. It was concluded that such pumps offered both optimal dimensions and a suitably simple overall design for the considered reactor parameters.

The report ends with a brief review of spccific hydraulic problems associated with these pumps, e.g. choice of diffuser design, inflow to the hydrostatic bearing, ctc. 


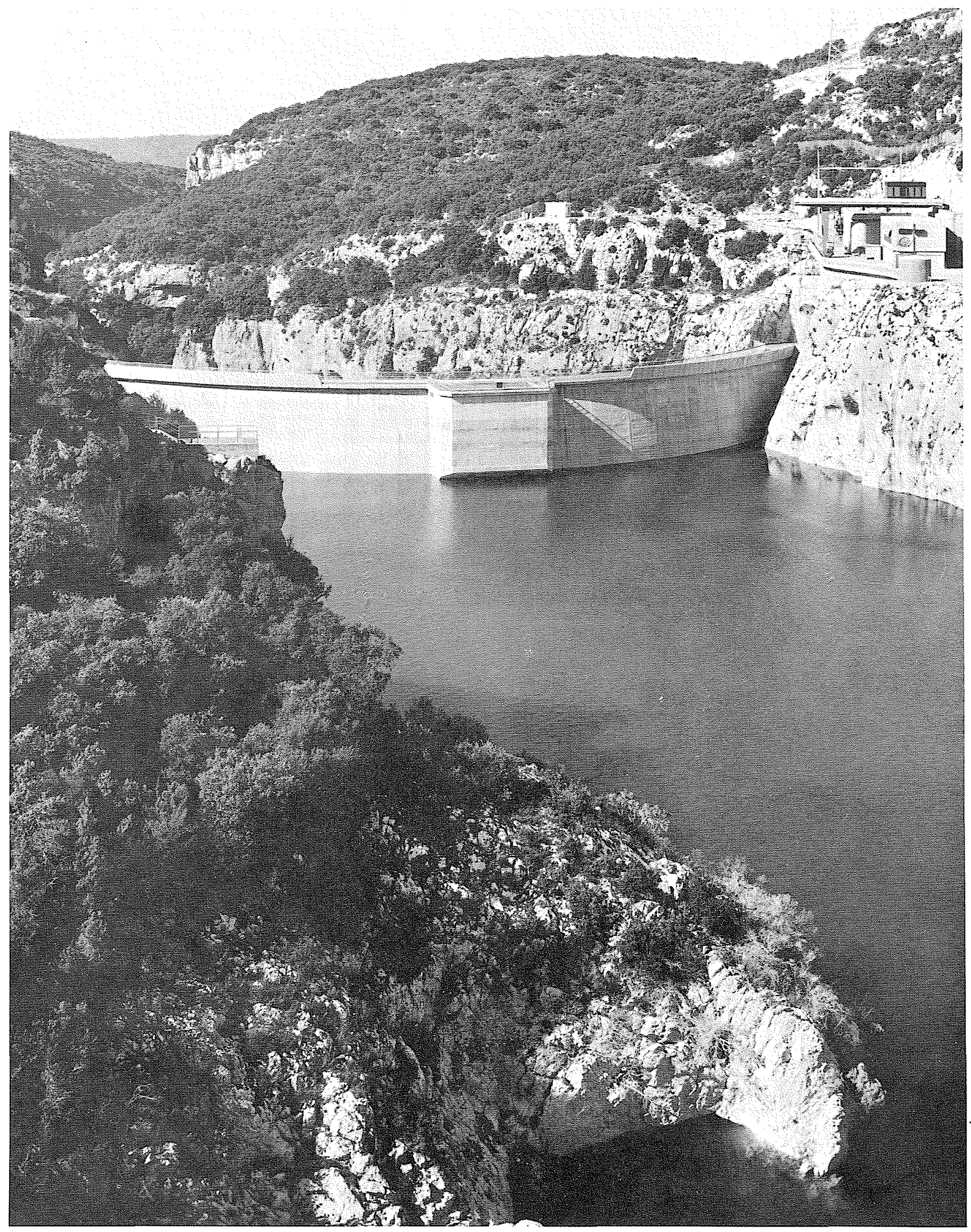

(Photothèque EDF - Pierre BERENGER) 